\title{
APRENDIZAJE COOPERATIVO HÍBRIDO Y SU EVALUACIÓN FORMATIVA Y COMPARTIDA EN EL GRADO DE EDUCACIÓN INFANTIL. UNA BUENA PRÁCTICA EN LA UNIVERSIDAD DE CANTABRIA
}

Hybrid Cooperative Learning and its Formative and Comparative Evaluation in the Degree of Children Education. A good practice in the University of Cantabria Aprendizagem Cooperativa Híbrida e sua Avaliação Formativa e Partilhada na Licenciatura de Educação Infantil. Boas práticas na Universidade de Cantabria

\section{Natalia González-Fernández (1) \\ Irina Salcines Talledo (2)}

(1) Facultad de Educación. Universidad de Cantabria, España. Teléfono: +34 942201270. Correo electrónico: gonzalen@unican.es

(2) Universidad de Cantabria, España. Teléfono: +34942201883. Correo electrónico: salcinesi@unican.es

\section{Resumen}

Se presenta una buena práctica de Aprendizaje Cooperativo y Evaluación Formativa y Compartida, desarrollada en la asignatura de Investigación e Innovación Educativa, en el Grado en Educación Infantil de la Universidad de Cantabria. Se trata de una experiencia que implica la apertura a relaciones dialógicas eficaces y de construcción cooperativa intra e intergrupos de estudiantes, así como la promoción de una cultura offline y online, en combinación con procesos cíclicos de evaluación formativa y compartida. En esta experiencia han participado 96 estudiantes de $4^{\circ}$ de Grado cuyas valoraciones han sido muy positivas, al incrementar su motivación, participación y autorregulación del aprendizaje. Generando un clima de aula en el que se contagiaban las ganas por seguir profundizando en el dominio de la materia.

Palabras clave: Evaluación formativa; evaluación compartida; aprendizaje cooperativo; grado educación infantil

\section{Abstract}

A good practice of Cooperative Learning and Formative and Shared Evaluation is presented, developed in the subject of Educational Research and Innovation, in the Childhood Education Degree of the University of Cantabria. It is an experience that 
implies the opening to effective dialogical relations and the cooperative construction of intra- and intergroups of students, as well as the promotion of an offline and online culture, in combination with cyclical processes of formative and shared evaluation. Ninety-six 4th Grade students have participated in this experience. Their evaluations have been very positive, increasing their motivation, participation and self-regulation of learning. Generating a classroom climate in which the desire to continue to deepen the mastery of the subject was contagious.

Keywords: Training assessment; shared assessment; childhood education degree; cooperative learning

\section{Resumo}

É apresentada uma boa prática de Aprendizagem Cooperativa e Avaliação Formativa e partilhada, desenvolvida na disciplina de Pesquisa e Inovação Educacional, na Licenciatura de Educação Infantil da Universidade de Cantabria. Trata-se de uma experiência que implica a abertura a relações dialógicas eficazes e de construção cooperativa intra e intergrupos de estudantes, bem como a promoção de uma cultura offline e online, em combinação com processos cíclicos de avaliação formativa e partilhada. Participaram nesta experiência 96 alunos do $4^{\circ}$ ano da licenciatura. As suas avaliações foram muito positivas, aumentando a sua motivação, participação e autoregulação da aprendizagem. Gerou-se um clima de sala de aula em que o desejo de continuar a aprofundar o domínio do assunto era contagiante.

Palavras-chave: Avaliação formativa; Avaliação partilhada; Aprendizagem Cooperativa; Licenciatura em Educação Infantil

\section{Introducción}

Según la UNESCO (2017), los sistemas educativos de todo el mundo sufrirán grandes modificaciones de aquí a 2030. La revolución tecnológica convertirá a las instituciones educativas en entornos interactivos en los que el docente orientará el aprendizaje.

Por otro lado, en la Guía Práctica para el cambio educativo en España (Instituto de Innovación en Negocios Educativos, 2016) reflexiona sobre cómo hacer frente a los cambios en el modelo tradicional de enseñanza y la adopción de nuevos enfoques de trabajo pedagógicos más acordes con el presente. En la citada guía, se trata de ofrecer 
una mirada introspectiva y crítica al ámbito educativo, que ayude a detectar los obstáculos, potenciar lo que se está haciendo bien y revertir las malas prácticas. Entre las soluciones que se proponen, se resaltan dos áreas: la metodología y la tecnología. En este contexto, los entornos de aprendizaje en línea ofrecen oportunidades para una mayor cooperación entre aprendices. Según Sáez López (2015), se están proponiendo procesos de enseñanza, aprendizaje y evaluación basados en la experimentación con entornos de aprendizaje online y herramientas que promueven la cooperación por pares o Peer-Led Team Learning (PLTL).

Se trata de modelos de enseñanza híbridos, dado que superan la necesidad de disponer y compartir espacios físicos entre estudiantes y, facilitan un proceso de aprendizaje que respeta los ritmos y características individuales. Actualmente, con la aparición de plataformas educativas, los entornos virtuales de aprendizaje juegan un papel positivo. Concretamente los entornos virtuales de aprendizaje (EVA o PLE), fomentan y facilitan el aprendizaje cooperativo (Tirado Morueta \& Martínez Garrido, 2010), a la vez que ofrecen flexibilidad temporal, posibilidad de recibir retroalimentación de compañeros y docentes, y aumento de motivación, consciencia y participación en el propio aprendizaje.

En el resumen del informe Horizón 2017 (Adams Becker et al., 2017), se define el aprendizaje cooperativo desde la perspectiva como una construcción social, que implica el desarrollo de actividades centradas generalmente en cuatro principios: el estudiante como protagonista del aprendizaje, énfasis en la interacción, trabajo en equipo, y desarrollo de soluciones a problemas reales. Aun teniendo en cuenta que esta tendencia se basa en la pedagogía, la tecnología juega un papel clave en su implementación. Por lo tanto, el aprendizaje cooperativo híbrido, combina la tecnología y la cooperación, permitiendo, tanto a estudiantes como docentes, trabajar en torno proyectos, al mismo tiempo que integran recursos online que, les permitirán expandir su aprendizaje.

\section{Contextualización}

En esta experiencia, han participado de forma constante y activa 96 estudiantes, se ha llevado a cabo en la Universidad de Cantabria durante el curso 2018-19 en el Grado de Educación Infantil. Concretamente, en la asignatura de Investigación e Innovación Educativa de $4^{\circ}$ curso, con una carga de 6 créditos ECTS. 


\section{Diseño y desarrollo}

A lo largo de los seis temas en los que se ha estructurado la asignatura, se han diseñado retos educativos grupales de dificultad progresiva, basados en la filosofía del Aprendizaje Cooperativo Híbrido y la Evaluación Formativa y Compartida. Concretamente, se ha desarrollado la práctica haciendo hincapié en la importancia de herramientas digitales para facilitar enfoques de aprendizaje cooperativo, ofreciendo plataformas para la comunicación (Google Drive, Blogs) y el desarrollo de actividades en entornos sincrónicos y asincrónicos.

Para entender mejor en qué consiste la metodología del aprendizaje cooperativo híbrido, se sintetiza en cuatro grandes pasos lo que Moll (2017), denomina "pilares básicos": (1) Organización de grupos y disposición del espacio en el aula. Se forman tres tipos de grupos: equipos de base, equipos esporádicos y equipos de expertos; (2) Implantación de la metodología cooperativa en el aula, teniendo en cuanta cinco aspectos fundamentales: dinámicas de cohesión que permiten a los estudiantes valorar el trabajo en equipo, las normas de funcionamiento que deben ser aceptadas por todos los miembros del grupo, los roles que cada estudiante lleva a cabo en su grupo, el plan y blog donde cada grupo base registra el trabajo y, por último, las exposiciones en las que cada grupo comparte, valora y coevalúa, el trabajo realizado; (3) Dinamización. Se proponen estructuras de aprendizaje en las que se inciden en objetivos y dinámicas concretas: "lápices al centro", "lectura compartida", "1, 2, 4", "rompecabezas" o "folio giratorio"; y (4) Evaluación, autoevaluación y coevaluación. Se diseñan rúbricas, que posibilitan la autoevaluación, coevaluación y heteroevaluación.

\section{Evaluación}

La evaluación de la buena práctica de aprendizaje cooperativo se realiza analizando las respuestas obtenidas en el cuestionario de autopercepción del aprendizaje cooperativo, cumplimentado por los estudiantes al finalizar la asignatura.

Hay que tener en cuenta que las actividades cooperativas específicas propuestas no han ido asociadas a una calificación numérica, con peso en la evaluación final.

Siguiendo la filosofía de la evaluación formativa, se han empleado como un sistema idóneo que favorece la mejora de los procesos de enseñanza, aprendizaje y evaluación, orientando y retroalimentando a los estudiantes para que, ellos mismos, reflexionen y 
emprendan acciones precisas que les permitan optimizar sus aprendizajes (RomeroMartín, Castejón-Oliva y López-Pastor, 2015).

\section{Conclusiones}

Los estudiantes valoran positivamente el aprendizaje cooperativo híbrido evaluado formativamente, debido al aumento de su motivación, participación, co-responsabilidad $\mathrm{y}$, desarrollo de habilidades sociales $\mathrm{y}$ emocionales. El feedback ofrecido por compañeros y docentes, a través de blog diseñados grupalmente es otro valor de la experiencia. Sin embargo, sigue habiendo grupos que no cooperan, y una vez finalizada la asignatura, no continúan alimentando las herramientas diseñadas para cooperar a lo largo de su vida profesional.

\section{Referencias}

Adams Becker, S., Cummins, M., Davis, A., Freeman, A., Hall Giesinger, C., \& Ananthanarayanan, V. (2017). The NMC Horizon Report: 2017 Higher Education Edition. Recuperado de: http://educalab.es/intef

Instituto de Innovación en Negocios Educativos. (2016). Guía práctica para el cambio educativo en España. Recuperado de: http://www.innedu.es/Guia-PracticaCambio-Educativo-en-Espa\%C3\%B1a_INNEDU_2016.pdf

Moll, S. (2017). Eduforics. Anticipando la educación del futuro: Las 10 mejores webs para iniciarse en el aprendizaje cooperativo. Recuperado de: http://www.eduforics.com/es/las-10-mejores-webs-iniciarse-aprendizajecooperativo/

Romero-Martín, R., Castejón-Oliva, F.J., \& López-Pastor, V. (2015). Divergencias del alumnado y del profesorado universitario sobre las dificultades para aplicar la evaluación formativa. RELIEVE, 21(1), 1-66. DOI:10.7203/relieve.21.1.5169

Sáez López, J.M. (2015). Metodología y tecnologías emergentes en contextos pedagógicos. En A. Medina Rivilla (Coord.) (2015). Innovación de la educación y de la docencia (pp.275-286). Madrid: Ramón Areces.

Tirado Morueta, R., \& Martínez Garrido, J.M. (2010). Creando comunidades virtuales de aprendizaje: análisis del progreso de las interacciones. Revista de Educación, $353,297-328$. 
UNESCO (2017). Informe de seguimiento de la educación en el mundo. La educación al servicio de los pueblos y el planeta: creación de futuros sostenibles para todos. París: UNESCO.

Recuperado

de: http://unesdoc.unesco.org/images/0024/002485/248526S.pdf 